\title{
Kafkas Blumfeld-Erzählung im Rahmen von Kafkas „Neutönertum“. Eine Entdeckung
}

„Wo keine Götter sind da walten Gespenster“ (Novalis in seinen Fragmenten).

„Das Adjektiv kafkaesk beschreibt ein unergründliches Gefühl der Bedrohung, der Unsicherheit oder des Ausgeliefertseins, etwa angesichts einer im Dunkeln liegenden Macht.

Der Begriff ... hat seinen Ursprung nicht in einer fremden Sprache, sondern im Namen des Schriftstellers Franz Kafka.

So sehen sich beispielsweise in Kafkas unvollendeten Werken Das Schloss und Der Process die Protagonisten einer undurchschaubaren und unerreichbaren Bürokratie ausgeliefert.

Zunächst wurde der Begriff nur im literarischen Umfeld verwendet, wo Werke, die eine ähnliche Grundstimmung ... wie die Werke Kafkas aufwiesen, als kafkaesk bezeichnet wurden. Im heutigen Sprachgebrauch können auch reale Situationen, Sachverhalte und Strukturen als kafkaesk beschrieben werden. "1 Soweit eine lexikalische Beschreibung des hier verhandelten Gegenstands. Es gibt, nebenbei gesagt, einige Kafka-Forscher, die bezeichnen das eigene Metier als kafkaesk. Der Verfasser dieses Essays zählt nicht zu diesen. Sein Bestreben geht eher dahin, das Kafkaeske selbst eine Strecke weit zu „entkafkaisieren“ - ohne dabei in so triviale Enthüllungen zu verfallen, dass alle Kafka-Forschung selbst als „kafkaesk“ zu bezeichnen sei. Dabei kommt einem, in einem allerdings selbst kafkaesken, mithin paradoxalen Zusammenhang, die immer noch brennend aktuelle Me-Too-Bewegung zur Hilfe. My Dark Vanessa

\footnotetext{
${ }^{1}$ So neueswort.de/kafkaesk im Jahre 2019. Die Hervorhebungen eben dort.
}

(C) Der/die Autor(en) 2021

B. Neumann, Umrisse einer Dritten Kultur im interdisziplinären Zusammenspiel zwischen Literatur und Naturwissenschaft, ELECTRISCHER PROMETHEUS. Umrisse einer Dritten Kultur im interdisziplinären Zusammenspiel zwischen Literatur und Naturwissenschaft, https://doi.org/10.1007/978-3-662-63204-8_17 
lautet der Titel eines aktuellen, zum Bestseller aufgerückten, sehr lesenswerten Buches, des Debut-Buchs der amerikanischen Autorin Kate Elisabeth Russel. Dessen plot dreht sich um die Frage, ob es „Liebe“ geben kann zwischen einem erwachsenen, sehr attraktiven, auch emotional intelligenten Mann in Autoritätsstellung, und einer erst 15 Jahre alten, zudem noch rothaarigen und literarisch ausserordentlich begabten Frau (deren Begabung allerdings erlischt, als sich die ehemals nymphenhaft Verführerische einsinnig als „Opfer“ zu begreifen lernt, unter brachialer Beihilfe einer amerikanischen Universität, die ihren Dozenten als einen "Schuldigen“ entlarvt und ihn am Ende in den Selbstmord treibt). Die Antwort, ob solches Geschehen noch „Liebe“ heissen kann, muss „Nein“ lauten in unseren Zeiten einer zuweilen hysterischen Gleichstellungsdebatte, wobei freilich die erhebliche Qualität des zur Rede stehenden Textes gerade in seinem intertextuellen Bezug auf Nabokovs Lolita darin liegt, als autonomes Kunstwerk die Parteinahme seiner Autorin zu korrigieren im Sinn der Herstellung einer paradoxalen Ausgewogenheit. Dieser Text erscheint mithin klüger als seine Autorin, wobei es möglich erscheint, dass diese um eben diesen Tatbestand wusste - und ihn schreibend einkalkuliert hat. Ein hoch intelligentes Buch also, das auch gar nicht daran vorbei kommt, dort, wo es ,wirklich“, also existentiell paradox wird, auf Kafka zu rekurrieren. Der attraktive, pädagogisch charismatische und sehr „männliche“ „Verführer“ dieser modernen Lolita soll zur Strecke gebracht werden von nicht nur einer, sondern gleich mehreren Aktivistinnen, die die eigene „Vergewaltigung“ durch ihn erfinden aus Gründen des „feministischen Kampfes“. Das „wahre Opfer“ sowie der „Verführer“ wissen um den Tatbestand, und der besondere Klingelton des Smartphones, der für den übergriffig gewordenen Pädagogen reserviert wurde im Smartphone der (im Bereich der sog. ,,sozialen Medien“ supermodernen) Lolita macht schliesslich folgenden Dialog möglich: „, „I know what's coming, “ he says. „I've taught there for thirty years and they'r tossing me out with the trash..."Well, they' re monsters”, (Vanessa, B.N.) says... It's like a goddam horror movie. „Sounds more like Kafka to me"", beschliesst danach die dunkle Vanessa diese Bestandsaufnahme. ${ }^{2}$ Ihr literarisch geschulter Geschmack erkennt die ästhetische und diagnostische Überlegenheit des „Kafkaesken“, was sich paradoxerweise auch daraus speist, dass sie am Ende selbst die falschen Anschuldigungen gegen ihren Geliebten unterstützen wird. Der bleibt als Selbstmordopfer zurück. Vanessa büsst zusammen mit ihrer Dunkelheit all ihre physische und intellektuelle

\footnotetext{
${ }^{2}$ Kate Elisabeth Russell, My Dark Vanessa, London 2020, S. 53.
} 
Brillanz ein. Am Ende, und der deutsche Leser fragt sich, spricht daraus die Weisheit des alten Theodor Fontane, oder eine dann ganz neue feministischkreatürliche Bescheidenheit der Einsicht, kommt es doch tatsächlich zum Kauf einer Hündin, und deren „Liebe“ soll ersetzen, was die Männer eh' nicht geben können: bedingungslose Hingabe. (Doch auch Kafkas Blumfeld-Erzählung war ja ursprünglich als Hunde-Geschichte gedacht: In diesem Sinne sind sowohl der Prager, wie auch die Amerikanerin schlussendlich ,,auf den Hund gekommen“).

Soviel also zum „Kafkaesken“ in den Zeiten von Me-Too, wobei eine Art höherer „englische“ oder sogar luziferische Gerechtigkeit in diesem Buch waltet, so, wenn der Verführer impotent wird, als er sich der nun bereits dreissigjährigen Vanessa noch einmal sexuell annähern will, durchaus mit deren Einverständnis. Begreift man diese Volte als amerikanische Spielform romantischer Ironie, gelangt man zurück zur Sache selbst, an deren Ausgangspunkt: Franz Kafka begriff sich wie gesagt als einen „Blutsbruder“ Heinrich von Kleists. Der Begriff steht zwar nicht wörtlich in seinen Tagebüchern, ist aber akzeptabel, wie ausgeführt. Erinnert er doch daran, dass die Niederschrift des Verschollenen u. a. durch die Rezeption eines Theodor-Körner-Films angeregt wurde, in dem ein strahlend weißes Pferd bestrickend mit den schwarzen, schmucklosen Uniformen der antifranzösischen Freikorps-Kämpfer kontrastierte, zu einem ikonengleichen Bild verschmolzen. Das noch frühe Kino machte aus dem Prager jenen „elektrischen Prometheus“" ${ }^{\text {, }}$ dessen gesamtes Romanwerk dann vom Gegensatz Dunkel-Hell geprägt erscheinen wird. Grelles, zuweilen aber noch „griechisches“ Licht im Erstling; verschneite, lastende, durchgehende Dunkelheit im abschließenden Schloss-Roman. Licht und Dunkel aber sind bereits im Mythos mit dem Blitz des Zeus konnotiert; seit dem 18. Jahrhundert auch mit Voltaismus und Galvanismus, ferner mit dem angesprochenen Mesmerismus, und danach mit Elektrizität, Maxwell'schem Elektromagnetismus und schließlich mit Einsteins Relativitätstheorien. Es geht immer um das (rätselhaft-widersprüchliche) Phänomen nicht-mechanischer Kraftentfaltung und Kraftübertragung als eines der allerersten, zum Mythos gewordenen Menschheitsrätsel. Darüber hinaus um ein in seiner Gesamtheit neues Weltbild, wie es sich bei Descartes, dann Newton und Leibniz, schließlich durch Einstein und die Quantenphysik herausbildete. Auf das Zeitalter des alles bestimmenden Säfteaustauschs (17.Jahrhundert) folgte

\footnotetext{
${ }^{3}$ Zum Begriff und seinem Umfeld Benjamin Specht, Physik als Kunst, Berlin 2010, S. 357, eine umfassende und hoch reflektierte Arbeit zu Frühromantik, Mesmerismus etc., die auf Kafka freilich nicht eingeht. Die Schreibweise ,electrisch“ wird immer dann verwendet, wenn es gilt, vor-Maxwell'sche, mithin noch romantische Denkweisen zu kennzeichnen.
} 
das der mesmeristischen „Electrizität“ samt des „tierischen Magnetismus“ (18. Jahrhundert). Mit diesem gelangte dann das gesamte noch ,mechanische“ Zeitalter Newtons und Leibniz' an sein Ende. Wurde zu Beginn des 20. Jahrhunderts abgelöst durch Einsteins Theorien - und erreichte dabei auch Kafka. Wie und wie intensiv dies geschah, wird im Folgenden beschrieben, als ein neu entdeckter, aufzudeckender Sachverhalt. Nun wurden sogar Zeit und Raum nicht mehr als absolute gedacht, - wobei auch schon früher der (elektrische) Funke des (frühromantischen) Genies in der Lage gewesen sein sollte, die hergebrachte Ordnung von Raum und Zeit zu sprengen. Das wiederum hatte nicht zufällig, und für unseren Zusammenhang sehr passend, mit Heinrich von Kleist zu tun. Der war bereits vor Kafka, aber naturgemäß noch nicht so „modern“ wie dieser, seinerseits ein ,elektrischer Prometheus“ gewesen.

Wo nun der Blick aufs Konkrete, u. a. auf, zu Unrecht als altmodisch verschriene, „Einflüsse“ zielt, gerät Franz Kafkas erst einmal eher verschnarcht anmutende Erzählung über einen „älteren Junggesellen“ namens „Blumfeld“ in den Fokus. Im Jahr 1915 über einen Prager Bürger geschrieben, der angesichts seines nunmehr besiegelten Junggesellendaseins auf zwei rätselhafte Bälle trifft, die sich - surreal, aber behäbig, absolut fremd und doch unmittelbar zur Stelle ohne jede Vorbereitung, schillernd und im Betragen doch „einfältig“, so bedrohlich wie belustigend, also insgesamt ,aus einer anderen Welt“ -- als Inbegriff des „Kafkaesken“ erweisen. Letzteres ein Tatbestand, der in der Kafka-Forschung nirgendwo bestritten worden ist. Mithin eine Reise ins Innerste des „Kafkaesken“ selbst? Freilich, im Herz dieser vermeintlichen Finsternis angekommen, zeigt sich Unerwartetes: Wir sind mitten in der (sogar atomar leuchtenden!) Herzkammer des 20. Jahrhunderts angelangt. Nämlich dort, wo aus dem vorigen „mechanischen“ Weltbild das der modernen Atomphysik entstand. Also ein seinerseits erst einmal sehr Dunkles, das allmählich erhellt zu werden vermochte und eine Präfiguration des hier im Dienste einer „Dritten Kultur“ Unternommenen. Deshalb müssen wir ein wenig weiter ausholen und, aber nur scheinbar, das Thema wechseln: In Franz Werfels Roman der Oper (1924), vom Wiener Autor dem sterbenden Kafka zugesandt und von diesem tatsächlich noch gelesen, im mühseligen Lesevorgang eines im "Sanatoryum“ (so die Schreibweise von Kafkas letzter Wegbegleiterin) an der Tuberkulose Sterbenden, findet sich ein jüdischer Deutscher, ein „neutönerischer“ (mithin à la Schönberg zwölftönerischer) Komponist namens „Fischböck“. Der Mann stammt - aus „Bitterfeld“. Also Franz Kafka als der am meisten radikale ,atonale Romankomponist“ innerhalb der damaligen Prosakunst, weit mehr radikal als zum Beispiel Thomas Mann oder eben Franz Werfel selbst? Es ist zwingend anzunehmen, dass Werfel den Doktor Faustus, bzw. den Zauberberg von Thomas Mann kannte, samt 
anderer Texte dieses Schriftstellers, und umgekehrt bei Kafka ähnliches voraussetzte. Der Zauberberg samt seiner Kapitel über die Einstein'sche Relativitätstheorie war 1924 erscheinen, freilich nach Kafkas Tod. Im Januar des Jahres 1947 dann hatte der Großschriftsteller Thomas Mann im kalifornischen Exil seinen Roman über Das Leben des deutschen Tonsetzers Adrian Leverkühn erzählt von einem Freunde beendet. Ein Künstlerroman, mit deutlichen Anklängen an das biographisch-geistige Schicksal des Friedrich Nietzsche, und der Versuch, den Nationalsozialismus literarisch zu „bewältigen“, nachdem dieser kapituliert hatte und der Holocaust als dessen innerstes Wesen unabweisbar geworden war. 55 Mio. Kriegstoten sollten mit einem Phänomen in Verbindung gebracht und somit ,aufgearbeitet“" werden, das dem Wagner-Adepten und Musikenthusiasten Thomas Mann samt seinem „symphonischen Erzählen“ ganz nahe war, womöglich näher als der endlich besiegte Menschheitsfeind Nationalsozialismus: Wie hält es der moderne Musikfreund und bürgerliche Künstler mit dem Atonalen? Unter diesem Aspekt hängen Kafka als „electrischer Prometheus“ und Thomas Manns Doktor Faustus so eng zusammen, dass sie als Fleisch vom eigenen Fleisch, Geist vom eigenen Geist erscheinen. Das ist hier buchstäblich zu nehmen: Der Teufel, das ,ist“ der auch unserem Text sattsam bekannte Theodor W. Adorno mitsamt seiner Entscheidung für die Zwölftonmusik. Hier gilt es genau zu lesen und sich mit anderen Lesarten, etwa der Rüdiger Safranskis in seinem Romantik-Buch, auseinander zu setzen. Insbesondere deshalb, weil Dank der Prominenz des beteiligten Personals: Tomas Mann und Theodor W. Adorno als den beiden Vertreter deutschen Schreibens und Denkens im 20. Jahrhundert, und beider Begegnung auch noch als moderne Teufelsbegegnung arrangiert, eine tendenziell weltweite Aufmerksamkeit garantiert war. Die Situation in Manns Roman, man erinnert sich, war folgende: Der fiktive Erzähler Zeitblohm findet Aufzeichnungen seines da bereits verstorbenen Komponistenfreundes Adrian Leverkühn über dessen Begegnung mit dem Teufel vor. Ein, von uns aus gesehen, tua res agitur wird vorbereitet durch das, was wiederum dieser Komponist direkt vor seiner lebensentscheidenden Begegnung unternommen hatte: Er „las Kierke-

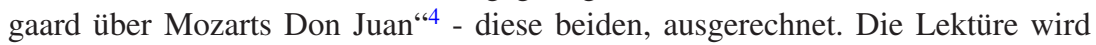
in Manns Roman weitergeführt als Schilderung einer rabiat realistischen Teufelsbegegnung, als wiederbelebtes Mittelalter im Ambiente des modernsten sonnenüberfluteten Kalifornien. Der Gottseibeiuns sitzt auf einem Rosshaarsofa im

\footnotetext{
${ }^{4}$ Thomas Mann, Doktor Faustus, in: Gesammelte Werke in dreizehn Bänden, Fischer Taschenbuch Verlag, Frankfurt/Main 1990, Bd. VI, S. 297 ff.
} 
Dämmerlicht, mitten im Raum, gar nicht verschämt; er wechselt spielerisch seine Gestalt, ohne daß der Kältestrom, der von ihm ausgeht, abflauen würde. Man spricht nicht nur deutsch miteinander, sondern „fein altdeutsch“, wobei der Besucher zunächst als ein „Strizzi. Ein Ludewig. Und mit der Stimme, ... eines Schauspielers" vorgestellt wird. Die Erscheinung jedenfalls artikuliert sich, ganz wie Adorno es im Leben tat, als ein geübter Redner mit Nasenresonanz. Und sie verwandelt sich, als es zum Thema der Schönberg'schen Atonalität kommt, noch einmal: „Hatt einen weißen Kragen um und einen Streifenschlips, auf der gebogenen Nase eine Brille mit Hornrahmen, ... eine Mischung von Schärfe und Weichheit das Gesicht: die Nase scharf, die Lippen scharf, aber weich das Kinn." Der noch junge Theodor W. Adorno ersteht vor uns, „bleich und gewölbt die Stirn, aus der das Haar wohl erhöhend zurückgeschwunden, aber von ders zu den Seiten dicht, schwarz und wollig dahinstand - ein Intelligenzler, der über Kunst, über Musik, für die gemeinen Zeitungen schreibt, ein Theoretiker und Kritiker, der selbst komponiert, soweit das Denken es ihm erlaubt.“" Mithin der Teufel als Adorno, oder eben auch: Adorno als Teufel. Und als wäre das nicht genug des fiktional erzeugten finstersten Mittelalters: Dieser Teufel trat durchaus gewinnend auf, selbst noch, als er über die „kalte“ Zwölftonmusik professoral geübten Vortrag hält. Dem Komponisten war es ,in seiner Gesellschaft wohler geworden“, „obschon die Kälte fortfuhr, mir zuzusetzen." 5 Leverkühns Gesprächspartner spricht originale Adorno-Sätze, mit nur ganz leichter, vom Autor stets kontrollierten parodistischen Überdrehung: „Die Subsumption des Ausdrucks unters versöhnlich Allgemeine ist das innerste Prinzip des musikalischen Scheins. Es ist aus damit. Der Anspruch, das Allgemeine als im Besonderen harmonisch erhalten zu denken, dementiert sich selbst." ${ }^{\circ}$

Die Atonalität also als einzige verbliebene musikalische Ausdrucksform in den Zeiten eines umfassend „falschen Lebens“. Gerichtet gegen jede Harmonie, vor allem gegen die der vormals phythagoräisch behaupteten der Himmelssphären. Nachdem Leverkühn, nun in Thomas Mann selbst verwandelt, sich noch die eigene Theorie der Parodie (etwa des „Bildungsromans“) von seinem Besucher hat absegnen lassen, vollführt dieser seine Metamorphose wieder zurück ins unverfälscht-mittelalterlich Diabolische. Er wird zu Mephisto, vollzieht darin ein quasi okkultes italienisches Jugenderlebnis seines Verfasser nach, wie wir sehen werden. Ein bebrillter Teufel trifft nun die entscheidende Absprache,

\footnotetext{
${ }^{5}$ Thomas Mann, Doktor Faustus, a. a. O., S. 317 f.

${ }^{6}$ Thomas Mann, Doktor Faustus, a. a. O., S. 318.
} 
vollzieht den Goethe'schen Pakt, mit Leverkühn als neuem Faust und unter der neuen Bedingung: „Du darfst nicht lieben.“7 Als ein „knapp behostes Mannsluder"8 verfügt der Mann'sche Teufel dies, und unter anderem dies Faktum führt uns hinein in die Auseinandersetzung mit der Safranski'schen Lesart des zur Rede stehenden nunmehr neuen Teufelspakts. Rüdiger Safranski hatte bemerkt: „Dass sich in der Gefährdung des Künstlers die Gefährdung der deutschen Seele spiegeln sollte, nahm man auch deshalb nicht ungern zur Kenntnis, weil es sich bei Leverkühn um einen genialen Künstler handelte, der zudem frappante Ähnlichkeit mit Nietzsche hatte; ein ganzer dämonischer Apparat aus syphilitischer Ansteckung und Teufelspakt wurde aufgeboten." ${ }^{\text {" }}$ Und doch war es in der Realität des Textes Zeitbloom, und nicht etwa Leverkühn, der das deutsche Bürgertum mit seiner Kriegsbegeisterung des Jahres 1914 samt Ablehnung der Münchner Räterepublik und Besuch intellektueller Zirkel aus Kosmikern, Apokalyptikern und Dezisionisten repräsentierte. Leverkühn hingegen verblieb durchaus Apolliniker. Als solcher dem rauschhaften Treiben der Reaktionäre vornehm fremd - was man nur schwer als „dionysisch“ verstehen kann, wie Rüdiger Safranski es tut, vorzüglich, um den von ihm behaupteten Tatbestand zu verteidigen: Dass nämlich Thomas Mann „Adornos Anregungen bezüglich der künstlerischen Profilierung Leverkühns gerne folgte. "10 Was stimmt - und doch nicht stimmt. Will sagen: Mir erscheint die Situation komplizierter; und ich meine entscheidende Hilfe bei hochkarätigen Spezialisten in Sachen Thomas Mann, Nietzsche, Wagner und Adorno gefunden zu haben. Danach stellt die Angelegenheit sich doch nuancierter dar: Gegen seinen (politischen) Willen hätte sich dann die Figur unter der Hand Thomas Manns verändert. Des nur noch ironisch ,auctorialen“ Autors ästhetische Abneigung gegen die Schönberg'sche Lehre war nämlich derart entschieden, dass man die neu gewonnene Komponistenüberzeugung seiner Figur eher so zu lesen angehalten ist: Leverkühn holt der Teufel, weil er sich mit der Zwölftonlehre Schönbergs überhaupt einlässt! Das meint: Thomas Mann war und blieb Wagnerianer, egal, welche Musik der „Führer“ geliebt haben mochte; und der Lübecker wusste das eigentlich selbst, wie sein genialer

\footnotetext{
${ }^{7}$ Thomas Mann, Doktor Faustus, a. a. O., S. 331.

${ }^{8}$ Thomas Mann, Doktor Faustus, a. a. O., S. 333.

${ }^{9}$ Rüdiger Safranski, Romantik, a. a. O., S. 566.

${ }^{10}$ Rüdiger Safranski, Romantik, a. a. O., S. 573.
} 
Essay Bruder Hitler es belegt. ${ }^{11}$ Daraus einen politischen „Vorwurf“ gegen Thomas Mann zu konstruieren, wäre freilich ebenso absurd, wie die darin enthaltenen Widersprüche aus falsch verstandener Loyalität nicht zu erwähnen. Dieser „Großschriftsteller“ als der bedeutendste deutsche Romanautor des 20. Jahrhunderts war eben ein „Deutscher“ - allerdings mitsamt seinem unabdingbaren Anspruch darauf, dass ,wirkliches Deutschtum“ seit Goethe immer auch die Überschreitung alles National-Bornierten in sich tragen müsse. Dieter Borchmeyer hat darüber geschrieben, davon gleich noch mehr. Thomas Mann blieb jedenfalls „deutsch“ auch in seinem amerikanischen Exil; war daneben „deutsch" aber auch in jenem Sinne, den Safranski analysiert hat: "Es fehlte in den ersten Jahren nach 1945 ein politisches Raisonnement, das nicht sogleich in die übergroßen Fragen auswich, ... das ein Gegengewicht hätte bilden können zu einem Geist, der oft entweder zu hoch oder zu tief ansetzte, beim Nichts oder bei Gott, beim Untergang oder beim Aufgang des Abendlandes."12

Auch Thomas Mann ging es bei seiner Leverkühn-Figur „um alles“, um „Nichts“ oder eben um „Gott“ (bzw. „Teufel“" in dessen ebenso „faustischer“ Verbindung zu Gott dem Herrn). Der Fachmann Adorno war ihm damals als „Berater“ höchst willkommen, als ein umfassend funkelnder Geist von wie immer etwas teuflischer Brillanz. Der exilierte Hüter der deutschen Kultur, als den Thomas Mann sich sah, gewährte dem philosophierenden Musik-Gelehrten sogar unerhörte Vorrechte, wenn er ihm das noch unveröffentlichte Manuskript des Faustus zur Durchsicht überliess, ein Entgegenkommen ohne gleichen in Thomas Manns gesamtem Schriftstellerleben, das seinen Gipfel darin fand, dass Adorno Thomas Mann jene musikalischen Einfälle diktierte, die dieser seinem neuen Faustus zuzurechnen wünschte - und dieser kooperationswillige Autor sah in dem Geburtshelfer seines Textes gleichwohl eine Luzifer-Verkörperung, sozusagen aus Gründen seines musikalischen Instinktes? Es scheint so. Dabei besass der paradoxale Tatbestand seine Gründe, die tief in die Geschichte nicht nur der Stadt München hinabreichten, sondern insgesamt in die des damaligen Übergangs der „Dichter-und-Denker“-Nation auf die Seite des Nationalsozialismus'. Er besass aber auch, davon gleich noch mehr, näherliegende Gründe in Machinationen der Familie, in von Tochter Erika und „Frau Thomas Mann“ (so Kaja Mann gelegentlich über sich selbst) durchgesetzten Herabminderungen der Rolle Adornos. Dieser Kompositionsschüler Alban Bergs und vormalige ein-

\footnotetext{
${ }^{11}$ In diese Richtung auch Hermann Kurzke, Thomas Mann. Das Leben als Kunstwerk, München 1999, S. 515.

${ }^{12}$ Rüdiger Safranski, Romantik, a. a. O., S. 575.
} 
flussreiche Frankfurter Musikkritiker hatte Thomas Mann im kalifornischen Exil ja nicht nur anregende, sogar agitatorisch begeisternde Bücher genannt und zum Durcharbeiten ins Haus gebracht. Sondern er hatte darüber hinaus den Kern des Romans (sowie den von Thomas Manns eigener, tiefster und lebenslanger Schreibproblematik) getroffen: Wie gelange ich zur imperativen Inspiration, zum end-gültigen Einfall, zum stetigen, durch keine Selbstrelativierung mehr gefährdeten Schreibantrieb? „Sehr wahrscheinlich hat diese Problematik Thomas Mann geholfen, das Dilemma seines modernen Faust auf den Punkt zu bringen: die Frage der Inspiration. Adorno hatte somit den richtigen Griff getan; er hatte ... den Zugang zu einem Diskurs eröffnet, der aufgrund seiner musiktheoretischen und künstlerpsychologischen Brisanz sehr zur Sache gehörte."13 Damit hatte Manns musikkundiger Helfer zudem die eigentliche Problematik der post-Wagner'schen Epoche deutscher Musik- und Philosophiegeschichte in ihrer Leitthematik getroffen (am Beispiel des Musiktheoretikers Hans Pfitzner übrigens, was hier nicht weiter entfaltet werden kann). Denn im Teufelsgespräch, das Adorno mit blitzender randloser Brille und professoraler Rhetorik bestreitet, darin faustisch die Bedingungen des sattsam bekannten Goethe'schen Paktes neu festlegend, ging es darum, den künstlerischen Schaffensakt als eine einerseits genial-unabhängig machende, also befreiende, aber andererseits eben auch rauschhaft verführerische (und darin giftige Abhängigkeit bewirkende) Konstituante zu bestimmen - die freilich das Dilemma aller Nachfahren Richard Wagners, nämlich das Epigonentum, zu bannen versprach. Durch eine geradezu gläubig-okkult verstandene Inspiration, die keinerlei Nachbesserung mehr erfordern würde, sollten ein immerwährender Durchbruch und unverdrossen revolutionärer Innovationsschub möglich werden, um die unbedingte Führerschaft im Geistigen, in der Musik zuoberst, verliehen zu bekommen. Aus solchem romantischen Erbe Richard Wagners heraus, und als instinktives Festhalten an dessen rauschhafter Tonalität ereignet sich in Thomas Manns Doktor Faustus die hier verhandelte epochale und so folgenreiche Luziferisierung des Theodor W. Adorno. „Wer diesem Phantom von inspirierter Genialität folgt, wird sich bedenkenlos der hier gleichsam lächelnd antizipierten ,Barbarei erdreisten'. Leverkühn folgt ihm und antizipiert darin jenen anderen, genialen' Führer, der seine Barbarei nicht bloss in Töne setzen wird, sondern sie in Blut und Eisen übersetzt." 14 Was bedeutet: Die Avantgarde, die Manns Leverkühn exekutieren

\footnotetext{
${ }^{13}$ Hans Rudolf Vaget, Salome und Palestrina als historische Chiffren, in: Wagner Nietzsche - Thomas Mann, Festschrift für Eckhard Heftrich, Frankfurt/Main 1993.

${ }^{14}$ Hans Rudolf Vaget, Salome..., a. a. O., S. 79.
} 
wird, scheint zwar dem Modell der Schönberg/Adorno'schen Neuen Musik verpflichtet; sie gründet aber in ihrem Tiefsten in einer vormodernen, wenn nicht sogar reaktionären und Wagner wie Pfitzer verpflichteten Künstlerpsychologie, die alle Kunst nur als „seliges Diktat“ aus dunklen Tiefen heraus und als welterobernde Genie-Tat zu begreifen vermag. „Eine solche Kunstauffassung, so legt dieser Roman nahe, arbeitet dem reaktionären Genie-Evangelium des Nationalsozialismus in die Hand. Das Hinter-die-Schule-Laufen, dessen sich Leverkühn am Ende zeiht und das auf das Verfehlte der deutschen Geschichte im Grossen verweist, ist demnach im Werdegang des scheinbare abseitigen Tonsetzers vorgezeichnet: der Rückfall in die Barbarei auf dem Weg nach vorn, die Verquickung von Romantik und Moderne im Geistig-Seelischen." 15

Eben diesem Syndrom war der Wagnerianer Thomas Mann weiterhin verpflichtet; er huldigte ihm freilich im Glauben, dass es per se nicht-chauvinistisch und europäisch-umfassend geprägt aufträte, eben als „tiefstes Deutschtum“ in weltbürgerlicher Übersteigung seiner selbst. ${ }^{16}$ So hatte der Lübecker es bei seinem grossen Vorgänger Goethe gelernt, so hat es dann auch Manns eigener Sohn als imaginierter Nachfolger seines Vaters begriffen. Klaus Mann, der bereits offen homophil lebende und seinerseits Nicht-Mehr-Wagnerianer hat geschrieben: „Dass das Thema der ,Verführung ' für Zauberer (also für Thomas Mann, B.N.) so charakteristisch - im Gegensatz zu mir. Verführungsmotiv: Romantik Musik - Wagner - Venedig - Tod - ,Sympathie mit dem Abgrund“."17 Manns „Luziferisierung“ Adornos mochte also durchaus instinktiver Abweisung entsprungen gewesen sein, doch der dann veröffentlichte Text des Romans konterkariert die Absicht seines Verfassers ganz streng: Luzifer mag der Teufel sein, aber er ist eben doch auch - der Lichtbringer. „Die von Adorno vermittelte Neue Musik übersteigt den autobiographischen Horizont des Romans, der aber dadurch zu einer das Persönliche transzendierenden Rechenschaft über den Weg der deutschen Kultur in die Barbarei gesteigert ist." ${ }^{18}$ (Wie lautet doch eines von Adornos Aperçus, das geradezu Sprichwort-Charakter errungen hat? Dass es immer die grossen Kunstwerke seien, die an ihren fragwürdigen Stellen „Glück“ hätten). Ferner: Der hochkarätige Thomas-Mann-Kenner Hermann Kurzke hat zu

\footnotetext{
${ }^{15}$ Hans Rudolf Vaget, Salome..., a. a. O., S. 81.

${ }^{16}$ Vgl. dazu ausführlich Dieter Borchmeyer, ,Ein Dreigestirn ewig verbundener Geister. Wagner, Nietzsche, Thomas Mann und das Konzept einer übernationalen Kultur, in: Festschrift für Eckhard Heftrich, Frankfurt/Main 1993.

${ }^{17}$ Klaus Mann, Tagebücher 1931-1949, Reinbek 1995 (unter dem Datum 4. 4. 1933).

${ }^{18}$ Hans Rudolf Vaget, Salome..., a. a. O., S. 82.
} 
diesem Komplex festgestellt: „Thomas Mann hat das faschistische Deutschland leidenschaftlich bekämpft und sich trotzdem tief mit ihm identifiziert. Er hat 1945 vom Teufelspakt Hitlerdeutschlands gesprochen und es im Bilde Fausts gesehen, des einsamen Denkers in seiner Klause, der aus Verlangen nach Weltgenuss und Weltherrschaft seine Seele dem Teufel verschreibt und dann, 1945, buchstäblich vom Teufel geholt wird." 19 Und der gesamte Komplex ist damit immer noch nicht ausgeschritten, wie immer seine Dimensionen in Kurzkes angeführter Publikation allerdings abgesteckt erscheinen. Dieser Komplex besass nämlich eine Vorgeschichte: Der noch junge Thomas Mann, in Italien zu Gast und nach seiner Entscheidung, sein Leben als Künstler zu verbringen (und vermutlich auch nach einem homosexuellen Erlebnis mit einem Strichjungen) hatte doch tatsächlich vermeint, den Teufel erblickt zu haben. „Die Teufelserscheinung hat einen ganz leisen okkultistischen Anklang. Einen ähnlichen Herrn auf dem Sofa will Thomas Mann damals selbst gesehen haben ... mit ... widrig knapper Hose und gelben, vertragenen Schuhen. ${ }^{20}$ Damit nicht genug. Es gab nämlich auch noch eine verstörende Nachgeschichte. Nach Erscheinen des Faustus-Romans kam es zu ernsten Verstimmungen zwischen Adorno und Mann. Adorno musste sich von Mann hintergangen fühlen, hatte doch die Familie des Epikers (mehr oder weniger hinter dessen Rücken zudem) durchgesetzt, dass Adornos Anteil an der Entstehung des Romans wahrheitswidrig herabgespielt werden sollte. Das endete nicht nur in bitterem Streit zwischen Mann und Adorno, der es für Mann dann nötig machte, die Entstehung des Dr. Faustus gesondert zu beschreiben, um Adorno doch noch Gerechtigkeit widerfahren zu lassen. Sondern auch noch in folgender Posse: Der „Neutöner“ Schönberg verlangte gerichtlich einen Anteil an Thomas Manns Tantiemen, sollte dieser doch in seinem Roman Schönbergs Neue Musik für seine, des Autors eigene Erfindung ausgegeben haben! Die so ertrag-

\footnotetext{
${ }^{19}$ Hermann Kurzke, Thomas Mann. Das Leben als Kunstwerk, München 1999, S. 514. Siehe in diesem Zusammenhang auch Thomas Manns Essay Deutschland und die Deutschen, in: Thomas Mann, Gesammelte Werke in dreizehn Bänden, Frankfurt/Main 1990 (Fischer Taschenbuch Verlag), Bd. XI, S. 1126.

${ }^{20}$ Hermann Kurzke, a. a. O., S. 501. Siehe dazu auch Thomas Manns selbstbiographischen Versuch On Myself, in: Thomas Mann, Gesammelte Werke in dreizehn Bänden, Fischer Taschenbuch Verlag, Frankfurt/Main 1990, Bd. XIII, S. 135 ff. Der gleiche Text übrigens auch im Kapitel In Schlangennot im Roman Joseph und seine Brüder, welche Doppelung die Gewichtung dieser Thematik für Thomas Mann nur noch einmal unterstreicht. In diesem Zusammenhang auch Thomas Manns Dem Dichter zu Ehren, in dem Kafkas Schloss als das Werk eines religiösen Satirikers gewürdigt wird, mit Akzentsetzung auf der Problematik der Assimilation und auch auf Kafkas besonderer Nähe zu Novalis.
} 
reiche Kooperation zwischen dem norddeutschen Epiker und dem hessischen Musiktheoretiker endete somit in einem höllischen Chaos zerbrochener Freundschaften und absurder juristischer Ansprüche, dem allenfalls noch das homerische Gelächter, das ihm eigentlich gebührte, zu endlicher Versöhnung verhelfen könnte. Und doch bleibt es hier wohl eher beim Höllengelächter, das ja bekanntlich auch Engelsstimmen umfassen soll?

Wir aber finden uns nach einem Umweg, der freilich direkt in unsere Ausgangsthematik zurückführte, erneut mit der grundlegenden Antinomie Tonalität-Atonalität konfrontiert. Der nach Franz Werfels Willen „Neutöner“ Franz Kafka, der allerdings die Sentimentalität, Naturversenkung und die psychologisch differenzierte „Beseelung“ noch gründlicher infrage gestellt hatte als Flaubert vor ihm in seiner berühmten Madame Bovary - er hätte also die Atonalität in die Romanliteratur eingebracht? Albert Einstein wiederum, ein laienhafter Virtuose der Geige und vormaliger Duo-Partner seines klavierspielenden Assistenten Ludwig Hopf, liebte das musikalische „Neutönertum“ ganz und gar nicht. Bei Thomas Mann konnte er eine ähnliche Aversion voraussetzen, ob er nun den Doktor Faustus gelesen hatte, oder auch nicht. Dadurch wird folgende Erzählung glaubhaft, wie sie über die beiden amerikanischen Exilanten (Mann fühlte sich freilich weniger in seinem kalifornischen Exil zu Hause, als der Internationalist Einstein in seinem an der Ostküste) in Santa Monica, in Manns Villa kolportiert wurde: Einstein habe Mann aufgesucht. Und ein Exemplar von Kafkas Schlo $\beta$ auf den Schreibtisch des Großschriftstellers gepfeffert, auf dem auch Manns Zauberberg lag (der seinerseits, wie erwähnt, eine kurze, von Castorp referierte Geschichte der Relativitätstheorie enthält). Bei diesem Rencontre habe der Physiker, unklar ist, ob mit Erinnerung an sein Prager Treffen mit Kafka, oder auch nicht, jedenfalls erklärt - das Kafka'sche Buch sei „pervers“. Wenn das stimmt (vermutlich, weil es stimmt), hat der Naturwissenschaftler gegenüber dem Schriftsteller sich auf dem Gebiet des literarischen Urteils, mithin auf dem Terrain der „Ersten Kultur“, so verhalten, wie auf dem der „Zweiten Kultur“ gegenüber den Quantentheoretikern Heisenberg und Bohr. "Gott würfelt nicht" hätte dann in beiden Bereichen gegolten. Der nicht mehr genügend ,auctoriale“ Verfasser Franz Kafka mitsamt seinem von Einstein inspiriertem letzten Roman wäre vom zuständigen (und hier dennoch inkompetenten) Physiker brachial auf den Stand des 19. Jahrhunderts zurückverwiesen worden. ${ }^{21}$ Ein epochales

\footnotetext{
${ }^{21}$ Siehe dazu das jüngst erschienene Buch von Michael D. Gordin, Einstein in Bohemia, Princeton \& Oxford 2020, S. 8. Ferner, als Originalquelle, Robert W. Stallman, A HungerArtist, in: Franz Kafka today, Madison 1958, S. 61.
} 
Missverständnis, bei dem die Reaktion Thomas Manns uns leider nicht überliefert ist. Beider geschildertes Zusammentreffen scheint dennoch glaubhaft, begründet in ihrem ganz besonderen Verhältnis zueinander. Sie waren in Manns Princetoner Zeit nicht nur Nachbarn gewesen und hatten einander häufig besucht. Hochachtung, Freundschaft, eine bei Mann seltene Hochschätzung von Sanftmut, Bescheidenheit, ja gewinnende „Kindlichkeit“ des anderen bestimmten das Bild. ${ }^{22}$ Es gibt einen Schnappschuss, der Thomas Mann im intensiven Gespräch mit dem Physikergenie zeigt. „So ganz einfach neben dem? Mir schwindelt“ - das hat der Schriftsteller als Widmung darauf geschrieben. ${ }^{23}$ Beide waren Emigranten, ehemals schlechte Schüler, nun aber überragende Grössen auf jeweils ihren Gebieten, mit Spass an Ironie und sogar Allotria. Man hatte aber auch politisch zueinander gefunden, etwa durch eine gemeinsames Ehrendinner mit dem amerikanischen Präsidenten Roosevelt, nach der Ehrenpromotion 1935 in Havard, beide übrigens vom FBI „listet" als angebliche „fellow travellers“ des kommunistischen Feindes. Das alles verband tief. Beide hatten seit den Tagen der Weimarer Republik als gutherzige Weltverbesserer agiert, mit einer langen Liste gemeinsam unterschriebener Petitionen und Denkschriften, von der Freiheit der Kunst bis hin zu Kinderheimen der Roten Hilfe. Im Oktober 1945 rufen sie gemeinsam zur Verhinderung eines Atomkriegs auf, die zentrale Einstein'sche Entdeckung, wonach Masse und Energie im Grunde dasselbe waren, hat der Physiker dem Dichter selbst erläutert, der hatte das alles im Zauberberg dargestellt, womöglich aber ohne wirkliches Begreifen. Für ihn war jedenfalls „Einstein“ zum Synonym für ,moderne Physik“ ebenso geworden, wie „Thomas Mann“ zum Synonym für „,moderne Literatur“ für Albert Einstein. Das berechtigte diesen schon, in Manns geheiligtes Arbeitszimmer einfach einzubrechen mit dem Verlangen, seines Kollegen Kafka Schloss zu rüffeln. Das alles macht jedenfalls die oben wiedergegebene Erzählung sehr glaubhaft. Noch 1955 hatte der Lübecker in seinem Versuch über Schiller den Satz geschrieben: „Wut und Angst, abergläubischer Hass, panischer Schrecken und wilde Verfolgungssucht beherrschen eine Menschheit, welcher der kosmische Raum gerade recht ist, strategische Basen darin anzulegen, und die die Sonnenkraft äfft, um Vernichtungswaffen frevlerisch daraus herzustellen." 24 Ob Albert Einstein freilich

\footnotetext{
${ }^{22}$ Derart beschreibt Thomas Mann seinen Nachbarn Einstein unter dem 31.10.1925 an Ida Harz, in: Die Briefe Thomas Manns, hrsg. v. Hans Bürgin und Hans-Otto Mayer, Frankfurt/ Main 1976.

${ }^{23}$ Bei Klaus W. Jonas, Über meine Thomas-Mann-Bibliografie, in: Der Wagen, Lübeck 1997/98, S. 152.

${ }^{24}$ Thomas Mann, Gesammelte Werke in dreizehn Bänden, a. a. O., Bd. IX, S. 950.
} 
diesen Satz kannte, und ob er ihn gut geheissen hätte, bleibt zweifelhaft. Doch beide planten noch im Jahre 1954 erneut eine Aktion gegen den drohenden Atomkrieg, Erika bricht im Auftrag ihres Vaters zu abklärenden Gesprächen nach London auf, man plante Grosses - doch wenig später ereilt Thomas Mann seine Krankheit zum Tode.

Hier noch eine abschliessende Zusatzbemerkung: Theodor W. Adorno befand sich damals ebenfalls im Exil, bei ihm lag das allerdings in New York, er war aber markant anwesend als teuflischer Fachmann fürs „Neutönertum“ in Manns Doktor Faustus, wie geschildert. Der Sozialphilosoph hat seinerseits sogar ähnlich „Perverses“ an Kafkas Texten bemerkt, das aber zustimmend-verständnisvoll. Wir folgen auch hier seiner Spur: "In solchen Exzentrisitäten folgt Kafka Freud, mit eulenspiegelhafter Treue, bis zum Absurden. Er entreißt die Psychoanalyse der Psychologie ... Die Person wird aus einem Substantiellen zum bloßen Organisationsprinzip somatischer Impulse. Bei Freud wie bei Kafka ist die Geltung des Beseelten ausgeschaltet", es träten die elektromagnetisch zu denkenden Impulse des gesellschaftlichen Feldes an dessen Stelle. ${ }^{25}$ Wenn dem so war, und dem war allerdings so, dann verfasste Kafka seine von Adorno charakterisierte Darstellung als jener aus Prag stammende Autor, der er gewesen ist. Denn es hatte sich in seiner Astronomen-Stadt ja so verhalten: Auch nachdem das geozentrische Weltbild überwunden war (durch u. a. Galilei), wirkte die griechische Idee einer „Sphärenharmonie“ weiter, und dies gerade im Prag der ausgehenden Renaissance. Damit stand die Moldaumetropole in bedeutenden Traditionen: Pythagoras von Samos hatte bereits in der griechischen Antike angenommen, dass ,der Kosmos eine durch mathematische Proportionen optimal geordnete Ganzheit sei und dass sich daher in der Astronomie dieselben Gesetzmäßigkeiten zeigen wie in der Musik. In übertragenem Sinn wird der Begriff ,Sphärenmusik' heute auch für die Übertragung von Proportionen aus der Astrophysik in musikalische Beziehungen verwendet." ${ }^{\text {"26 }}$ Mithin die interoder auch transdisziplinäre Verbindung von Astronomie, Musik und Physik im Rahmen einer noch harmonischen Weltordnung. In unserem Zusammenhang (der Romancier Kafka als Prager „Neutöner“) erscheint einschlägig, dass Johannes Kepler seinerseits geglaubt hatte, die pythagoräische Tradition (zusammen mit Tycho Brahe) erneuern zu können, indem er sie auf sein heliozentrisches Modell

${ }^{25}$ Theodor W. Adorno, Aufzeichnungen zu Kafka, in: Prismen. Kulturkritik und Gesellschaft, Frankfurt/Main 1955, S. 311.

${ }^{26}$ https://de.wikipedia org./wiki/Sphärenharmonie S. 1 . 
übertrug. In der Weltharmonik von 1619 hatte Kepler seine Vorstellung von einem harmonisch geordneten Kosmos vorgelegt. Er trachtete danach, die Idee der Sphärenharmonie im Rahmen des damaligen Kenntnisstands über die Planetenanordnung und -bewegung neu $\mathrm{zu}$ formulieren. Wollte demonstrieren, dass die Gesetze der Astronomie im Prinzip dieselben seien wie diejenigen der Musik und der Physik. Das alles gehörte spätestens von da an zum Komplex „Musik, Physik und Astrologie im Kontext der Kosmos-Ordnung“, wie er für den Prager Kafka und seinen so musiknahen Freund Brod als selbstverständlicher Wissenshorizont angenommen werden muss. Dies erst bestimmt den Rahmen für Werfels zitierte Annahme, dass man in Kafka den ersten ,atonalen“ (und darum modernsten) Romancier erblicken könne. Wobei klar sein muss: Die komplizierten, weil unhintergehbar transdisziplinären Weltbild-Überlegungen in Kafkas Prag bildeten ihrerseits die Matrix für Franz Kafkas Schreiberexistenz. Deren Verständnis ist unterkomplex, also ohne Kepler, Einstein (und dann auch noch Heisenberg), gar nicht zu haben. Dies ein zwingendes Argument für die Konstituierung einer „Dritten Kultur“, was bereits ausgeführt wurde. ${ }^{27}$

Solche Feststellung gilt generell, und wurde auch bereits von Adorno in Bezug auf Kafkas Schreiben formuliert: „Aber gerade als hermetisches hatte es teil an der literarischen Bewegung des Dezenniums um den ersten Krieg, deren einer Brennpunkt Prag war und deren Milieu das Kafkas.“28 Der Befund vermag hier durch einige weitere Beispiele belegt zu werden: Etwa Gustav Meyrinks Prag-Roman Golem gehört ebenfalls in diesen Zusammenhang. Bei Rilke findet Ähnliches im Malte statt, und bei diesem Prager evoziert die Eule, die sich an der Sphinx reibt, ebenfalls die Assoziation des Sternenhimmels als eine Art Hallraum vergangener Harmonik. Letztere ist immer weniger zu haben, je weiter die Moderne fortschreitet. Nicht nur die menschliche Erfahrungswelt, das gesamte Universum beginnt "schwarze Löcher" zu bekommen, inzwischen liegen diese ja sogar optisch wahrnehmbar vor. Gerade deren Existenz hatte man in der magischen Moldau-Stadt mit ihren „Durchhäusern“ immer schon vermutet. Denn erst die Löcher und Durchblicke in unserer Erfahrungswelt zeigen uns, was hinter der täglich erfahrenen Wirklichkeit liegt. Diese möglicherweise als „magisch" klassifizierte (und durchaus auch "Einstein'sche“, davon gleich) „Relativität“ in ein romansignifikantes System gesetzt, bringt die besondere „kafkaeske“ Atonalität neuester Wirklichkeitserfahrung hervor. Nicht

\footnotetext{
${ }^{27}$ Vgl. dazu die einleitenden Kapitel zur Konstituierung einer „Dritten Kultur“.

${ }^{28}$ Theodor W. Adorno, Aufzeichnungen zu Kafka, a. a. O., S. 326.
} 
zufällig wurde Sigmund Freuds Traumdeutung der Öffentlichkeit im gleichen Jahr zugänglich wie Arthur Schnitzlers unerhörte „Innerer-Monolog“-Novelle Leutnant Gustl (beide im Jahr 1900) - nur mit dem entscheidenden Unterschied, dass Kafka so nüchtern erzählt, als sei der sich entfaltende, politisch bereits präfaschistische Albtraum die unspektakuläre bürgerlich-altösterreichische Wirklichkeit selbst. Was sie ja auch war. Ferner: Anstelle des so weiten, „natürlichen“ und emotional bestimmten Felds der Liebe zeichnet Kafka objektiv begrenzte Feldlinien innerhalb eines gesellschaftlichen Energienetzes, das durch die attrattiva von Beamten bestimmt wird („Kakaniens“ „,chinesische“ Besonderheit bis zum Schluss, sie hatte, wie gezeigt, auch Ernst Weiss berührt). Ganz ähnlich wie dann beim anderen bedeutenden Modernen Michel Houellebecq flottieren die individuellen „Elementarteilchen“ zwischen den verschiedenen sozialen Magnetpolen hin und her, unter anderem von ,erotischen“ Motiven befeuert, also ganz so, wie im Schloss Frieda dem Beamtenhäuptling Klamm verfällt. Ist dies nicht auch ein Grund dafür, dass sich Klamm, Bürgel und die anderen hohen Beamten im Schloss sogar in ihrem Aussehen verändern, je nachdem mit wem sie es zu tun haben, und welche Erwartung sie gerade zu erfüllen haben? Bereits Theodor W. Adorno hat dies so gesehen, ohne an Einstein oder Heisenberg auch nur zu denken: ,... die gebannten Menschen handeln nicht von sich aus, sondern als wäre ein jeglicher in ein magnetisches Feld geraten. ${ }^{29}$ Eben. Erst in der neuen Atomphysik, nicht zuvor bei Newton und dessen Leibniz-Welt, und auch nicht in der Welt des „Realistischen Romans“ wurde jene ,moderne“ Veränderung in der Umlaufbahn der einzelnen Partikel möglich, die ihrerseits mit der hergebrachten Weltordnung beispielsweise noch bei Kepler unvereinbar gewesen war. Die Auflösung der alten Weltordnung war ihrerseits schrittweise erfolgt. Vom Philosophen Berkeley bis zu Wilhelm Dilthey, und anfangs auch noch bei Niels Bohr und Werner Heisenberg, bis man das Neue in den (erst mathematischen, dann mehr und mehr experimentellen) Griff bekam, wurde solche grundlegende „Auflösung der Ordnung" gefürchtet - bis sie dann ihrerseits zur neuen Ordnung der Dinge geriet. Das Schönberg'sche „Neutönertum“ liquidierte mit der Zwölftonmusik die griechisch hergebrachte, in der Renaissance dann erneuerte Harmonie in der Musik. Die neue Atomphysik tat dies physikalisch mit der Kosmos-Vorstellung von Newton und Leibniz’. Der „Innere Monolog“ (zuerst bei Schnitzler) und danach die „erlebte Rede“ (bei Kafka) ersetzten die objektive, noch

\footnotetext{
${ }^{29}$ Theodor W. Adorno, Aufzeichnungen zu Kafka, a. a. O., S. 329. Daran wären auch alle Dramatisierungen Kafkas gescheitert, denn: „Drama ist nur so weit möglich, wie Freiheit“ noch existiert.
} 
ungebrochen ,auctoriale“ und objektive Erzählperspektive des „Realistischen Romans“. Überall entstand Neues. Das nunmehr obsolet gewordene harmonische Weltbild war eine grundlegende Konstante auch für den „Realistischen Roman“ gewesen. Dessen ,auctoriale Erzählhaltung“ ein bestimmender Reflex eben noch der Newton-Welt, und dies alles im Bunde mit der bei Kepler dargelegten Harmonie-Vorstellung. Die Welt, der Kosmos noch als fest gefügte Sphärenharmonie, ohne jeden sozialen Bahnwechsel, mit dem (katholischen) Monarchen im Zentrum, um diese zentrale Einsicht noch einmal abschließend hervorzuheben. Was hier aufgezählt wurde, galt gerade für das „Kakanien“ in den Zeiten seines Untergangs. Kafkas Prag war in seinem Kultursektor ohne jeden Zweifel eine noch durchgehend österreichisch dominierte Metropole; und das womöglich mehr, als sie selbst es realisieren konnte unter dem politischen Druck der neuen Nationalbewegungen, deren Führer Tür an Tür mit Franz Kafka lebten, und die ihrerseits Wissen und politische Vorstellungen nicht selten an der KaiserWilhelm-Universität in Berlin bezogen hatten, oder eben an der nicht zufällig „,deutsch“ geheißenen Universität in Prag selbst, mit der dann Einstein selbst kollidieren wird, wie darzustellen noch aussteht.

Deshalb ist es entscheidend, die sozial- oder kulturhistorische Perspektive mit ins Bild zu holen. In der realen ,,kakanischen“ Gesellschaft war eine fortschreitende Assimilation der ghettobewohnenden Juden, ihr „Bahnwechsel“ hinein in die Hochfinanz und den bürgerlichen Handel, noch zu Maria Theresias Zeiten undenkbar gewesen. Erst Maria Theresias Nachfolger, ihr Sohn Joseph II., der Betreiber einer ,katholischen Aufklärung“, bewirkte dies. Kafka wusste um den Tatbestand. Der Romanschreiber lebte ja bereits in der liberalen Moderne, und sah sich mit der Einsicht konfrontiert, dass die Newton-Leibniz'sche Welt scheinbar ungestörter Harmonie gesellschaftlich ebenso hinunter gegangen war, wie physikalisch, musikalisch oder eben auch literarisch. Dieser kulturhistorische Tatbestand machte den Romanautor vor allem im späten Schloss-Roman zum „Neutöner“. Die hierin angelegte Parallele bewährt sich bis in Einzelheiten hinein. Neueste Physik nach Einstein, in der Quantenphysik, besagt weiterhin, dass solche „modernen“ Bahnwechsel nur dann zu registrieren sind, wenn die Partikel sie gerade vollziehen. Nur dann senden diese ihr Licht aus. Folgt man der Analogie, registriert man, dass in Kafkas letztem Roman etwa die Wirtin, der Bote Barnabas oder der Beamtenhäuptling Klamm immer dann hervorstechen bzw. überhaupt erst deutlich werden, wenn sie ihre Position im Spannungsfeld der Assimilation markant verändern. Als die Wirtin Frieda beschützen will, verfällt sie K. gegenüber in rabiaten Antisemitismus, wird zum Hindernis für K.'s weiteres Fortkommen, indem sie die Beine samt Rock breit aufspannt, damit auch realiter zum Hindernis werdend. Folglich gilt: Aller "moderner" 
Bahnwechsel der Protagonisten verändert seinerseits die dadurch nicht mehr „prästabilisierte“ Harmonie der bestehenden Gesellschaft. Auf diese Weise fielen also die Entdeckungen der modernsten Physik, weiterhin der neuesten Musiktheorie, und die gesellschaftlichen Umwälzungen in der damals beginnenden, ihrerseits „allermodernsten Moderne“ zusammen. Das geschah primär in den urbanen Zentren Wien und Prag, in deren Cafés und literarischen Gesellschaften samt akademischen Diskussionszirkeln. Auf der anderen, der Subjektseite, musste der assimilierte Jude per excellence Franz Kafka notwendigerweise für das revolutionäre physikalisch-gesellschaftliche (und, wie diskutiert, auch musikalische) Potential der Einstein'schen Relativitätstheorien besonders anfällig sein. Die darin enthaltene Auflösung der physikalisch-musikalisch-ordnungspolitischen Harmonie machte den Prager erst zum „Neutöner“ auf dem Sektor des modernen Romans, - so konnte jedenfalls der Musik- wie Literaturkenner Franz Werfel es wahrnehmen, wie geschildert. Ohne die Bekanntschaft mit Einsteins Theorie folglich kein „Neutönertum“ Kafkas? So wie ja auch, ein Seitenblick auf die Epoche der Körpersaft-Erklärungen sei erlaubt, wie sie Albrecht Koschorke so überzeugend beschrieben hat, der spätere Mesmerismus und die auf ihn wesentlich später folgende Atomphysik als eine „Fortsetzung“ der zuvor geltenden physiologischen Kommunikationstheorie qua Körpersaft verstanden werden kann. „Wenn im Prozess der Aufklärung neue Techniken und Mythen des Zirkulierens entstehen, so gehorcht der Übergang selbst noch dem Muster einer kompensatorischen Ökonomie zwischen den Flüssigkeiten“30 - die erst danach durch die kommunikative Macht des „tierischen Magnetismus“, und später dann die des elektromagnetischen Felds ersetzt werden sollte. Nunmehr geheimnisvoll unsichtbare Elektrizität anstatt treibender, drückender, sichtbar schwellender Säfte. Das scheinbar Jenseitige elektrischer Fernwirkung anstelle der alten Metaphysik, wobei beides seine ,poetische Verwirklichung“ jeweils in der Metapher hatte finden können. Hans Blumenberg hat dies beschrieben, in seiner phänomenalen Metaphorologie und ferner in Arbeit am Mythos, wir sind darauf bereits eingegangen.

\footnotetext{
${ }^{30}$ Albrecht Koschorke, Körperströme und Schriftverkehr. Mediologie des 18. Jahrhunderts, München 1999, S. 98.
} 
Open Access Dieses Kapitel wird unter der Creative Commons Namensnennung 4.0 International Lizenz (http://creativecommons.org/licenses/by/4.0/deed.de) veröffentlicht, welche die Nutzung, Vervielfältigung, Bearbeitung, Verbreitung und Wiedergabe in jeglichem Medium und Format erlaubt, sofern Sie den/die ursprünglichen Autor(en) und die Quelle ordnungsgemäß nennen, einen Link zur Creative Commons Lizenz beifügen und angeben, ob Änderungen vorgenommen wurden.

Die in diesem Kapitel enthaltenen Bilder und sonstiges Drittmaterial unterliegen ebenfalls der genannten Creative Commons Lizenz, sofern sich aus der Abbildungslegende nichts anderes ergibt. Sofern das betreffende Material nicht unter der genannten Creative Commons Lizenz steht und die betreffende Handlung nicht nach gesetzlichen Vorschriften erlaubt ist, ist für die oben aufgeführten Weiterverwendungen des Materials die Einwilligung des jeweiligen Rechteinhabers einzuholen.

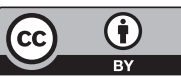

